\title{
Improvement of Vitamin $A$ and Trace Elements in Children with Anemia
}

T. DING*AND Q. JIANG

Department of Pediatric, Zhejiang Xiaoshan Hospital, No. 728 Yucai Road (north), Xiaoshan, Hangzhou, 311202, China

Ding and Jiang: Vitamin A and Trace Elements in Children with Anemia

In order to study the improvement of vitamin $A$ and trace elements in children with anemia, children whose serum vitamin $A$ is lower than $0.7 \mu \mathrm{mol} / \mathrm{l}$ or hemoglobin is lower than $115 \mathrm{~g} / \mathrm{l}$ were selected as research subjects and were divided into the iron supplementation group, the vitamin A supplementation group and 
www.ijpsonline.com

the control group (only nutrition education). Serum vitamin A was determined using high performance liquid chromatography. Hemoglobin was measured by semi-automatic hematology analyzer. Serum ferritin and serum transferrin receptor were measured using enzyme-linked immunosorbent assay. Serum vitamin A, hemoglobin, serum ferritin, serum transferrin receptor, prevalence of vitamin A deficiency and anemic changes were compared and analyzed before and after the treatment. The results showed that the serum vitamin A, hemoglobin and serum transferrin receptor levels in the group supplemented with vitamin A were significantly higher than those recorded prior to starting treatment. The level of serum ferritin, vitamin A deficiency and anemia were significantly lower after the treatment. The levels of hemoglobin and serum transferrin receptors in the group supplemented with iron were significantly higher after the treatment while serum ferritin level, vitamin A deficiency decreased significantly after the treatment. Although the level of vitamin A in serum increased but this increase was not significant, while the levels of all these indicators in the control group remain unchanged after treatment.

Key words: Vitamin A, trace elements, childhood anemia, hemoglobin, serum ferritin

Anemia (anemia) is a common clinical symptom that the volume of erythrocyte in human peripheral blood decreases below the lower limit of normal range ${ }^{[1]}$. Because the measurement of red blood cell volume in peripheral blood is very complicated, hemoglobin $(\mathrm{Hb})$ concentration is often used to express it in clinic $^{[2]}$. Anemia usually causes dizziness, fatigue, drowsiness, loss of appetite, paleness, vomiting, diarrhea, stomatitis and gastritis ${ }^{[3]}$. Anemia might harm multiple systems of the body in children including the digestive system, which leads to indigestion, loss of appetite; the nervous system leading irreversible damage to intelligence; low immunity, retardation of development and so on ${ }^{[4]}$.

Vitamin A, also known as retinol or anti dry eye vitamin, is a kind of alicyclic unsaturated monools with retinol biological activity, which can be divided into vitamin A1 and vitamin A2 from animal food sources ${ }^{[5]}$. Vitamin A has important physiological functions in human body, mainly for maintaining the normal visual function, maintaining the health of epithelial cells, promoting the synthesis of immunoglobulin, maintaining the growth and development of bone, promoting the growth and proliferation of cells, inhibiting the growth of tumors and acting as a nutritional supplement ${ }^{[6]}$. If vitamin A deficiency occurs, it would cause dry skin, scaling, alopecia, respiratory tract infection, dry eyes, photophobia, tears, blurred vision, lighter skin color, wrinkles, abscesses and other symptoms ${ }^{[7]}$. Trace elements are a group of minerals whose content is less than $0.01 \%$ of body weight, such as iron, zinc, copper, manganese, chromium, selenium, molybdenum, cobalt and fluorine ${ }^{[8]}$. Although their content in the human body is very small, these are indispensable for maintaining some important metabolic functions in the human body. Once the lack of trace elements occurs, the human body would become disease-prone and even endanger the safety of life ${ }^{[9,10]}$. Iron is one of the main components of hemoglobin and iron deficiency anemia results by the lack of iron. Lack of zinc will cause redness, papules and eczema in the mouth, eyes, anus or vulva. Lack of iron, copper and zinc will reduce the body's immunity making it prone to bacterial infection and even lead to death $^{[11]}$.

In conclusion, in order to understand the factors affecting children's anemia in more detail and improve their physical fitness, this study was taken up enrolling anemic children as the research subjects, to initiate nutritional intervention and explore the improvement of vitamin $\mathrm{A}$ and trace element levels in anemic children, so as to provide clinical evidence for improving children's anemia.

One hundred and fifty children with general anemic status reporting to Zhejiang Xiaoshan Hospital from August 2013 to July 2018 were randomly selected. Serum vitamin A and hemoglobin were measured in these children aged 3-6 y. Children whose serum vitamin $\mathrm{A}$ is lower than $0.7 \mu \mathrm{mol} / \mathrm{l}$ or hemoglobin is lower than $115 \mathrm{~g} / 1$ were selected as subjects. These children were randomly assigned to 3 groups, the iron supplementation group (50 children) who received iron supplementation, $60 \mathrm{mg} / \mathrm{w}$ ferrous sulfate tablets (Shenzhen Rock Biochemical Technology Co., Ltd., China) for $20 \mathrm{w}$; the vitamin A supplementation group (50 children) who received $50000 \mathrm{IU} / \mathrm{w}$ of vitamin A (tablets, Dalian Aquatic Pharmaceutical Co., Ltd., China) for $20 \mathrm{w}$ and the control group (50 children) 
who received only nutrition education. There was no statistical difference among the three groups with reference to age, sex and other basic data. An informed consent was signed by the patients or their family members and this study protocol was approved by the medical ethics committee of Zhejiang Xiaoshan Hospital.

The clinical symptoms of the children met the diagnostic criteria of iron deficiency anemia in children as recorded in the 3rd edition of the Standards for Diagnosis and Therapeutic Effect of Hematological Diseases were included. The compliance was good and the parents of the children signed the relevant informed consent. Exclusion criteria included while iron deficiency anemia was found in the children, serious organ diseases such as heart, brain, lung and kidney were found through examination. Patients with mental disorders, drug allergies, abnormal results of hemoglobin electrophoresis and children with severe iron deficiency anemia were excluded.

About $2 \mathrm{ml}$ of venous blood was collected from all children, the serum was separated within $24 \mathrm{~h}$ and stored at $-20^{\circ}$. The whole blood was centrifuged at $4000 \mathrm{rpm}$ for $10 \mathrm{~min}$. The supernatant was aspirated $(200 \mu \mathrm{l})$ and added to $200 \mu \mathrm{l}$ absolute ethanol (Shanghai Zhenyu Biotechnology Co., Ltd., China). The mixture was shaken, $1000 \mu \mathrm{l}$-hexane (Shanghai Chutai Chemical Technology Co., Ltd., China) was added mixed well and centrifuged at $4000 \mathrm{rpm}$ for $10 \mathrm{~min}$. Five hundred microlitres of the supernatant solution was separated and dried under nitrogen in a $37^{\circ}$ water bath. A mobile phase of methanol:water (90:10) was prepared and used in the chromatography. The detection wavelength was $313 \mathrm{~nm}$, the flow rate was $1.4 \mathrm{ml} / \mathrm{min}$, and the retention time was $5.06 \mathrm{~min}$. Adrop of venous blood from children was taken for anticoagulation and hemoglobin $(\mathrm{Hb})$ determination on a F-800 semi-automatic hematology analyzer (Sysmex Corporation, Japan).

The standard, quality control serum and test samples were added to a 96-well plate. Enzyme conjugates were added to each well and mixed evenly and kept in a refrigerator $\left(2-8^{\circ}\right)$. After $30 \mathrm{~min}$, the liquid from 96-well plate was discarded and washed 5 times with lotion. Liquid was aspirated and $50 \mu \mathrm{l}$ each of colour developer solutions A and B were added to each well. After mixing evenly the plate was kept in the dark at room temperature. After $15 \mathrm{~min}, 50 \mu 1$ of the terminating solution was added and mixed evenly. A microplate reader (Molecular Devices, USA) was used to measure the absorbance at $450 \mathrm{~nm}$ and the concentration of the sample was calculated.

The diluent of serum transferrin receptor reaction was added to 96-well plate with a sample of $100 \mu \mathrm{l}$. Standard, quality control serum and the test samples were added to each well. The mixture was shaken and incubated at room temperature. After waiting for $1 \mathrm{~h}$, the liquid from 96 well plate was discarded and it was washed 5 times with lotion. The liquid was aspirated, $100 \mu \mathrm{l}$ of colour developing solution was added to each well. After mixing evenly, it was kept at room temperature in the dark for $30 \mathrm{~min}, 100 \mu \mathrm{l}$ of terminating solution was added and mixed. The absorbance was measured at $450 \mathrm{~nm}$ with an automatic enzyme label and the concentration of the sample was calculated.

According to the standard of the second National health and nutrition Examination survey (NHANES II) the normal vitamin A level in serum (NVA) should be higher than $1.05 \mu \mathrm{M}(30 \mu \mathrm{g} / \mathrm{dl})$, serum levels of 0.7-1.05 $\mu \mathrm{M}(20-30 \mu \mathrm{g} / \mathrm{dl})$ would be defined as the marginal vitamin A deficiency (MVAD) and serum levels lower than $0.70 \mu \mathrm{mol} / \mathrm{l}(20 \mu \mathrm{g} / \mathrm{dl})$ is considered vitamin A deficiency (VAD). Both VAD and MVAD are with insufficient vitamin A (IVA). According to WHO and UNICEF criteria, children with hemoglobin less than $110 \mathrm{~g} / \mathrm{l}$ at age of 6-59 mo and children aged 5-11 y with hemoglobin less than $115 \mathrm{~g} / \mathrm{l}$ are regarded anemic. Among those, hemoglobin level between 90 to $110 \mathrm{~g} / \mathrm{l}$ is mild anemia, 60 to $90 \mathrm{~g} / \mathrm{l}$ is moderate anemia and below $60 \mathrm{~g} / \mathrm{l}$ is severe anemia.

SPSS21.0 statistical software was used for data processing and analysis. Quantitative data conforming to normal distribution are described by mean \pm standard deviation. Two independent samples $t$ test or rank sum test were used to compare the 2 groups. Chi-square test was used to compare values of different groups.

Results are shown in fig. 1A. The serum vitamin A in the group of vitamin A supplementation increased significantly $((p<0.05)$ after the treatment compared to that before starting the treatment. Although the serum vitamin $\mathrm{A}$ in the iron supplementation group increased to a certain extent but not significantly. The serum vitamin A in the control group was not different from that before the treatment.

Changes of hemoglobin $(\mathrm{Hb})$ in the 3 groups of children before and after the treatment are shown in fig. 1B. Compared to the level before treatment, the hemoglobin of iron supplementation group and vitamin A supplementation group increased significantly 
$(\mathrm{p}<0.001)$ post treatment, while the hemoglobin of control group remain unchanged.

Changes of serum ferritin (SF) in the 3 groups of children before and after treatment are shown in fig. 1C. Compared to the initial levels, the SF level in the iron supplementation group and the vitamin A supplementation group was significantly $(p<0.001)$ decreased, while the SF level in the control group was not significantly different from that before the treatment.

Changes of serum transferrin receptor (sTfR) in the 3 groups of children before and after treatment are shown in fig. 1D. Compared to the pretreatment levels of the sTfR in iron supplementation group and vitamin A supplementation group were significantly $(\mathrm{p}<0.001)$ increased. The sTfR levels in the control group were similar to those before the experiment.

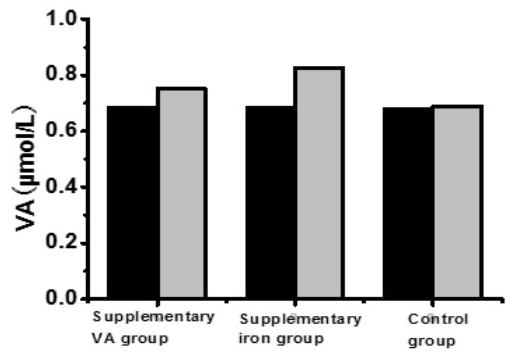

A

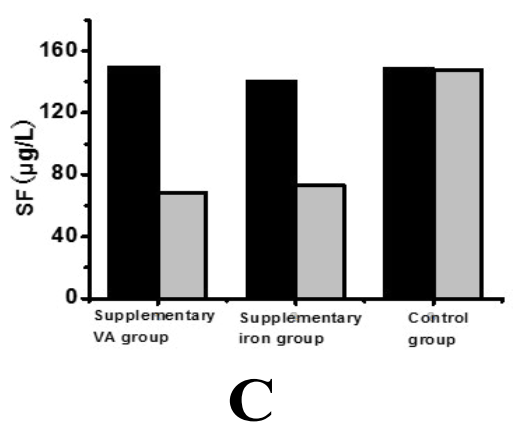

Changes of prevalence of vitamin A deficiency and anemia in the 3 groups of children before and after the 3.5 mo treatment period are shown in fig. 2. Compared to the levels before treatment, the prevalence of vitamin A deficiency and anemia in the iron supplementation group and vitamin A supplementation group are significantly $(p<0.001)$ lower. The prevalence of vitamin A deficiency and anemia in the control group did not change with treatment.

Serum vitamin A, hemoglobin, serum ferritin, serum transferrin receptor, prevalence of vitamin A deficiency and anemic levels were compared and analyzed before and after the treatment. The results showed that the serum vitamin A, hemoglobin and sTfR in the group supplemented with vitamin A were significantly higher than those levels before starting the treatment, and the

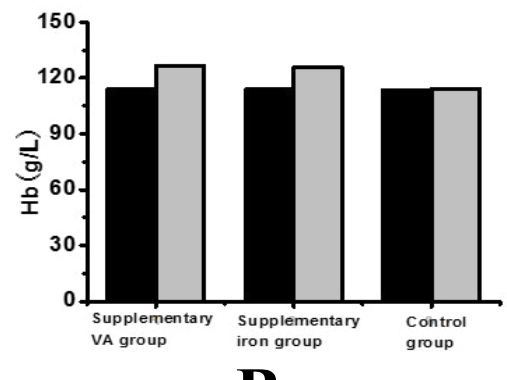

B

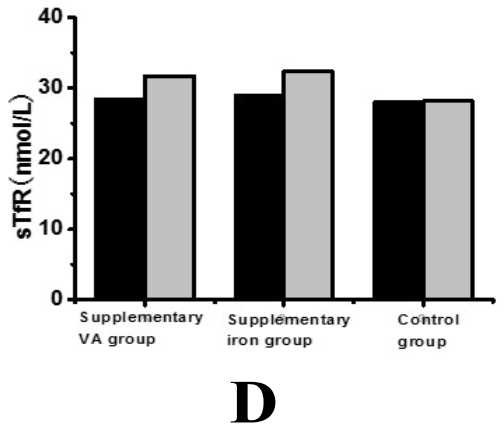

Fig. 1: Comparison all parameters measured in the 3 groups of children before and after treatment

A. serum vitamin A, B. hemoglobin, C. serum ferritin, D. serum transferrin receptor, ( $\square$ ) before and ( $\square$ ) after treatment
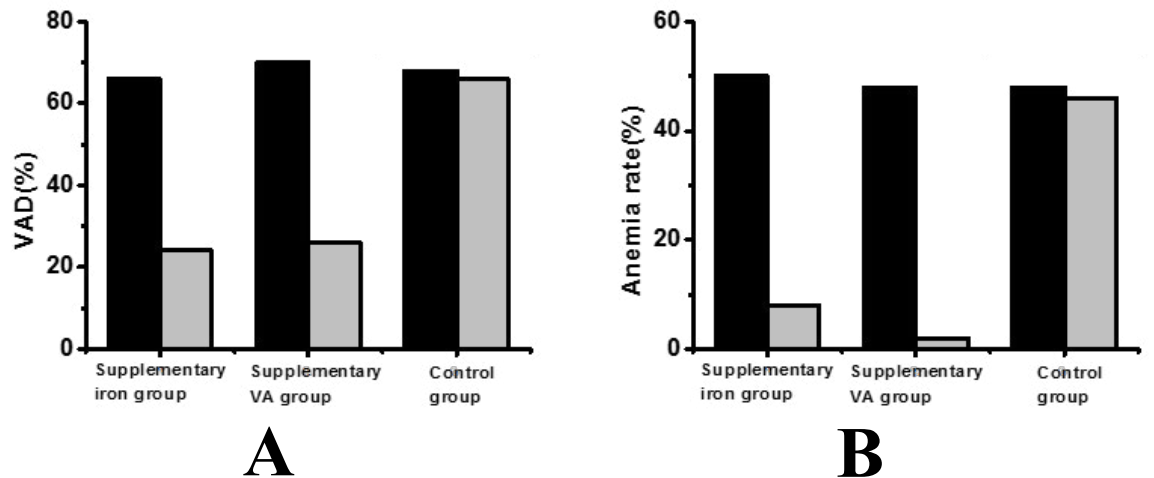

Fig. 2: Vitamin A deficiency and anemia in the 3 groups of children before and after treatment A. prevalence of vitamin A deficiency and B. rate of anemia ( $\square)$ before and $(\square)$ after treatment 
serum ferritin level, vitamin A deficiency and anemia were significantly lower after the treatment. The levels of hemoglobin and sTfR in the group supplemented with iron were significantly higher after treatment while serum ferritin levels and vitamin A deficiency were also changed. Anemia and the vitamin A deficiency decreased significantly treatment. The level of vitamin A in serum increased but not significantly, while all indicators in the control group remain unchanged.

Therefore, through the study on the improvement of vitamin $\mathrm{A}$ and trace elements in anemic children, it was observed that the serum vitamin A level in the iron supplementation group of children did not significantly increase, but changes observed in hemoglobin, serum ferritin, sTfR, vitamin A deficiency and anemia were similar to those observed in the vitamin A supplementation group, all of which were significantly improved. The study indicated that supplementation of $60 \mathrm{mg}$ iron and $50000 \mathrm{IU}$ vitamin A per week could improve anemia in children. However, the main shortcoming in this study appears to be smaller data size which would not allow to make firm recommendations and it is necessary to increase sample and data size in future research to generate more reliable data.

\section{REFERENCES}

1. Keller A, Ängquist L, Jacobsen R. A retrospective analysis of a societal experiment among the Danish population suggests that exposure to extra doses of vitamin A during fetal development may lower type 2 diabetes mellitus (T2DM) risk later in life. Br J Nutr 2017;117(5):731-6.

2. Cobelo-García A, Morán P, Almécija C. Historical record of trace elements (1983-2007) in scales from Atlantic salmon (Salmo salar): Study of past metal contamination from a copper mine (Ulla River, NW Iberian Peninsula). Chemosphere 2017;188:18.

3. Bahadir A, Erduran E, Değer O, Birinci Y, Ayar A. Augmented mitochondrial cytochrome c oxidase activity in children with iron deficiency: a tandem between iron and copper?. Arch Med Sci 2018;14(1):151-6.

4. Giménez-Forcada E, Vega-Alegre M, Timón-Sánchez S.
Characterization of regional cold-hydrothermal inflows enriched in arsenic and associated trace-elements in the southern part of the Duero Basin (Spain), by multivariate statistical analysis. Sci Total Environ 2017;41:s593-594:211226.

5. Bussan DD, Ochs CA, Jackson CR, Anumol T, Snyder SA, Cizdziel JV. Concentrations of select dissolved trace elements and anthropogenic organic compounds in the Mississippi River and major tributaries during the summer of 2012 and 2013. Environ Monit Assess 2017;189(2): 73.

6. Bahizire E, Bahwere P, Donnen P. High Prevalence of Anemia but Low Level of Iron Deficiency in Preschool Children during a Low Transmission Period of Malaria in Rural Kivu, Democratic Republic of the Congo. Am J Trop Med Hygiene 2017;97(2): 489.

7. Rzymski P, Ganz T. Comment on "serum hepcidin and soluble transferrin receptor in the assessment of iron metabolism in children on a vegetarian diet". Biol Trace Element Res 2018;185(2):1-3.

8. Golmaei M, Kinnarinen T, Jernström E, Häkkinen A. Efficient separation of hazardous trace metals and improvement of filtration properties of green liquor dregs by hydrocyclone. J Cleaner Production 2018;183:162-171.

9. Orsucci D, Briegel HJ, Dunjko V. Faster quantum mixing for slowly evolving sequences of markov chains. Computer Sci 2018;116(8):1185-94.

10. Dziegielewska-Gesiak S, Fatyga E, Kasiarz G, Wilczynski $\mathrm{T}$, Muc-Wierzgon M, Kokot T. Vitamins B12 and D deficiencies and macro- and microelement disturbances among diabetic elderly patients. J Biol Regul Homeost Agents 2019;33(2):47783.

11. Luo RM, Zhang XM, Du ZL. Haploidentical hematopoietic stem cell transplantation in the treatment of acquired aplastic anemia in children: a single center retrospective analysis. Zhonghua Chin J Pediatr 2018;56(7):529-533.

This is an open access article distributed under the terms of the Creative Commons Attribution-NonCommercial-ShareAlike 3.0 License, which allows others to remix, tweak, and build upon the work non-commercially, as long as the author is credited and the new creations are licensed under the identical terms

This article was originally published in a special issue:
Special issue on "Drug Development and Human Health in
China"
Indian J Pharm Sci 2020:82(1)spl issue2;101-105

